\title{
Surgery of the gallbladder
}

\author{
Hans-Jörg Mischinger
}

Diseases of the gallbladder are the most frequent in the gastrointestinal tract and account for most hospital admissions due to abdominal discomfort worldwide. In most cases gallbladder stones are diagnosed. According to the European Association for the Study of the Liver (EASL), nearly $20 \%$ of Europeans have gallstones and nearly half of them develop symptoms over their lifetime [1]. This results in approximately 900,000 gallbladder surgeries in Europe performed annually [2, 3]. In Austria nearly 19,000 patients are operated on their gallbladder yearly [4]. Preoperative management often reveals diseases wider than gallstones alone. These findings are frequently misdiagnosed and occasionally lead to uncertainty in the correct management or are only diagnosed intraoperatively as incidental findings. In the presented issue of the journal, pre-, peri-, and intraoperative findings and problems are addressed and discussed with respect to the current literature. Pathways are elaborated to develop pre- and intraoperative policies to overcome boundaries in gallbladder surgery.

The first article by Imamovic et al. discusses alternative approaches for critically ill patients besides surgical treatment of the gallbladder as well as surgical treatment in case of critically ill patients. With the high operative risk, surgical treatment often becomes less favored due to the rather high perioperative mortality and, therefore, alternatives such as percutaneous drainage, CT-guided drainage of the gallbladder, or other interventional therapies have been proposed in the literature for these patients.

\section{Univ.-Prof. Dr. H.-J. Mischinger $(\bowtie)$}

Klinische Abteilung für Allgemeinchirurgie,

Universitätsklinik für Chirurgie, Medizinische Universität

Graz, Auenbruggerplatz 29, $8036 \mathrm{Graz}$, Austria

hans.mischinger@medunigraz.at
Mischinger et al. address standardized concepts in gallbladder surgery. Establishing the "critical view of safety" is the only way to properly identify vessels and cystic structures and so prevent intraoperative biliary lesions. Anatomical landmarks are established, and anatomical variants are discussed as "bail out strategies" in case of intraoperative changes that hinder the establishment of the critical view and the identification of the important structures.

The complicated cholecystectomy is something that everyone has heard of, but the term itself still lacks standardized definition. Függer et al. try to establish the diagnoses which can lead to more complicated cholecystectomies requiring more sophisticated surgical skills. Surgical plans to avoid complications in these cases are discussed. He draws the conclusion that clinical decisions should follow a pathway based on patients' risks, favoring laparoscopic cholecystectomy whenever possible, and recommends the implementation of an institutional pathway to deal with difficult gallbladders.

Ammann et al. pose the problem of expertise in open cholecystectomies. Nearly $90 \%$ of all gallbladder removals are done laparoscopically and young surgeons thus often lack the expertise to perform open cholecystectomies. This is crucial, especially in risky situations, as gallbladder removal has to be performed openly as salvage therapy to bail out from critical laparoscopic situations. From this point of view, alternative patient management and treatment concepts should be considered to maintain patient safety.

Preoperative diagnosis before cholecystectomy has to be performed thoroughly, as not every thickening of the gallbladder wall is cholecystitis. Öfner et al. analyze the polypoid pathologies of the gallbladder and stress the indication for gallbladder removal. Although the risk of a malignant polyp is related to size, it remains uncertain how many polyps ultimately progress 
and become cancerous. Studies suggest surgery in case of polyps $>1 \mathrm{~cm}$. Asymptomatic polyps smaller than $10 \mathrm{~mm}$ do have the capacity to become cancerous and removal is recommended for risk factors such as PSC, solitary, and sessile polyps, starting from a size of $5 \mathrm{~mm}$. He concludes that no studies have been conducted to assess the adherence and effects of following these guidelines.

Wagner et al. elaborate algorithms for early gallbladder cancer as well as pre- and postoperative treatment options. If a carcinoma in situ (Tis) or a carcinoma confined to the mucosa (T1a) is diagnosed postoperatively, cholecystectomy is sufficient. If higherclass tumors are diagnosed (above $\mathrm{T}$ stage $1 \mathrm{~b}$ ), reoperation and liver resection with lymphadenectomy is performed to improve patients' prognosis.

This issue gives an overview of the management of complex and complicated gallbladder diseases and discusses various therapeutic options, including surgical as well as non-surgical approaches.

I sincerely hope you enjoy your journey through the upcoming pages.
Conflict of interest H.-J. Mischinger declares that he has no competing interests.

\section{References}

1. EuropeanAssociationfor theStudyoftheLiver(EASL). EASL Clinical Practice Guidelines on the prevention, diagnosis and treatment of gallstones. J Hepatol. 2016;65:146-81.

2. Aerts R, Penninckx F. The burden of gallstone disease in Europe. Aliment Pharmacol Ther. 2003;18(Suppl):49-53.

3. https://ec.europa.eu/eurostat/statisticsexplained/index. php?title=Surgical_operations_and_procedures_statistics\& oldid=502541\#Number_of_surgical_operations_and_pro cedures. Accessed: 10 May2021.

4. Statistik Austria. Spitalentlassungsstatistik, Entlassungen aus allen Krankenanstalten. 2012. https://www. statistik.at/web_de/statistiken/gesundheit/stationaere aufenthalte/medizinische_leistungen/index.html. Accessed: 10 May 2021.

Publisher's Note Springer Nature remains neutral with regard to jurisdictional claims in published maps and institutional affiliations. 